\title{
Prévalence de la pyospermie et retentissement sur la qualite du sperme chez les hommes infertiles.
}

\author{
L. AMMAR-KESKES ${ }^{1}$, N. CHAKROUN-FKI ${ }^{1}$, F. BOUZID ${ }^{2}$, A. BAHLOUL $^{3}$,

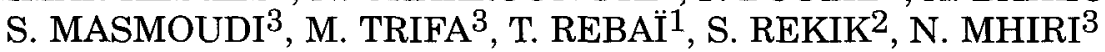 \\ ${ }^{1}$ Laboratoire d'Histologie-Embryologie et de Biologie de la Reproduction, Faculté de Médecine de Sfax \\ 2 Service de Gynécologie-Obstétrique, CHU Hédi Chaker, Sfax \\ 3 Service d'urologie, CHU Habib Bourguiba, Sfax.
}

\section{RESUME}

Il existe de nombreuses controverses concernant la relation entre la présence de leucocytes dans le sperme et la fertilité masculine. Dans le but de déterminer le retentissement de la pyospermie sur la qualité du sperme, nous avons entrepris une étude rétrospective dans laquelle nous avons comparé les paramètres du sperme ainsi que la fréquence des anomalies spermatiques entre des patients infertiles présentant au spermogramme une pyospermie (leucospermie $\geq 1 \mathrm{million} / \mathrm{ml}$ de sperme) et des patients non pyospermiques ( leucospermie < 50.000/ml de sperme). 833 spermogrammes réalisés selon le procédé standardisé de l'OMS ont été inclus dans l'étude. L'identification des leucocytes était réalisée par la technique de Endzt, basée sur la révélation de la peroxydase dans les granulocytes.

La prévalence de la pyospermie était rare $(5,88 \%)$. Nous n'avons pas trouvé de différences significatives entre les valeurs moyennes des principaux paramètres du spermogramme en présence ou en absence de pyospermie. Il n'existait aucune corrélation significative entre les valeurs de la leucospermie et les paramètres du sperme. Cependant, la morphologie était anormale dans seulement $34,1 \%$ des spermes avec pyospermie, contre $47,2 \%$ des spermes sans pyospermie $(p<0,05)$, ce qui pourrait traduire un rôle favorable des leucocytes dans l'élimina- tion des spermatozoïdes anormaux. L'oligospermie était au contraire plus fréquente dans le groupe de patients pyospermiques $(40,8 \%$ contre $20,3 \%, p<0,01)$. Ce résultat appuie l'hypothèse selon laquelle l'inflammation associée à la pyospermie entraînerait des remaniements histologiques aboutissant à une réduction de la perméabilité des voies génitales ou encore à une perturbation de la fonction gamètogène. Aucune relation n'a pu être établie entre la leucospermie et l'infection génitale, du fait de l'insuffisance d'arguments cliniques et biologiques. Une étude prospective comportant une exploration exhaustive est nécessaire afin de d'établir avec précision le lien existant entre la leucospermie et l'infection et déterminer le retentissement de la pyospermie sur l'activité des différents compartiments glandulaires du tractus génital masculin.

Mots clés : Pyospermie, leucospermie, spermogramme, spermatozoüde, infertilité masculine, inflammation.

Pour toute correspondance, écrire au Dr Keskes Leïla, Laboratoire d'Histologie, Faculté de Médecine, Avenue Majida Boulila, 3028, Sfax TUNISIE. 


\section{INTRODUCTION}

Il est admis que la présence des leucocytes dans le sperme est le reflet d'un état inflammatoire chronique non spécifique des voies génitales, associé parfois à une infection du tractus génital.

Depuis quelques années de nombreux arguments scientifiques enrichissent le débat concernant la relation entre la présence de leucocytes dans le sperme et la fertilité masculine. Cette relation reste l'objet de controverse , puisque de nombreuses études ont conclu à une altération de la qualité du sperme en présence d'un nombre élevé de leucocytes (pyospermie) [31, 12, 34, 35]; d'autres au contraire ont exclu tout retentissement de la pyospermie sur les paramètres du sperme [32,14].

Dans le but de contribuer à éclaircir la véritable relation existant entre la leucospermie et les paramètres spermatiques et de déterminer le retentissement de la pyospermie sur la qualité du sperme, nous avons entrepris une étude rétrospective portant sur des spermogrammes réalisés dans le cadre d'un bilan d'infertilité du couple. Nous avons comparé les paramètres du sperme ainsi que la fréquence des anomalies spermatiques entre les patients pyospermiques (leucospermie $\geq 1 \mathrm{million} / \mathrm{ml}$ de sperme) et les patients avec une leucospermie faible $(\leq 50.000$ leucocytes par millilitre de sperme).

\section{MATERIELS ET METHODES}

Notre étude a porté sur 833 spermogrammes pratiqués sur une période de 4 ans chez des hommes infertiles âgés de 36 ans en moyenne avec des extrêmes allant de 21 ans à 64 ans. La majorité d'entre eux $(66 \%)$ avait un âge compris entre 31 et 40 ans et $75 \%$ consultaient pour infertilité primaire d'une durée moyenne d'environ 6 ans.

1. Spermogramme : le recueil de sperme était effectué au laboratoire par masturbation après un délai d'abstinence sexuelle moyen d'environ $4,5 \mathrm{j}(2-10 \mathrm{j})$. Après liquéfaction, un spermogramme détaillé était fait selon le procédé décrit précédemment [3].

Dans cette étude les paramètres analysés étaient : le volume du sperme, la mobilité (exprimée en pourcentage de spermatozoïdes mobiles), la numération, la vitalité (déterminée par le test de Williams à l'EosineNigrosine), la morphologie (étudiée sur un frottis de sperme coloré par le Schorr) et la leucospermie. Cette dernière était déterminée par la technique cyto-enzymologique de Endtz utilisant la benzidine-cyanosine qui détecte les granulocytes en révélant l'activité peroxydasique contenue dans leurs granulations. Celles-ci apparaissent colorées en brun. La technique consiste à incuber $(\mathrm{V} / \mathrm{V})$ pendant 30 minutes un échantillon de sperme avec un réactif composé d'alcool $96^{\circ}$, de benzidine, de cyanosine ou phloxine $B$, d'eau distillée et d'eau oxygénée. Le comptage des granulocytes est fait sur cellule de Thoma.

Pour tous les paramètres analysés, nous avons considéré normales les valeurs conformes aux normes établies par l'OMS en 1992 [4].

En fonction des valeurs de la leucospermie, nous avons distingué deux groupes de patients: le groupe G1 avec une leucospermie $\geq 1$ million/ml de sperme (pyospermie) et le groupe G2 avec une leucospermie $\leq 50.000 / \mathrm{ml}$ de sperme. Nous avons alors comparé les données du spermogramme entre les deux groupes de patients et cherché d'éventuelles corrélations entre la leucospermie et les paramètres du sperme.

2. Méthode statistique : L'étude statistique était double : descriptive ( calcul de moyennes \pm écart-types et de la distribution des fréquences) et analytique avec comparaison des moyennes (test de student) et des pourcentages (test de Chi 2), et calcul du coefficient de corrélation $r$ (test de regression). La signification est donnée aux valeurs de $\mathrm{p}<5 \%$.

\section{RESULTATS}

Les valeurs moyennes des différents paramètres du spermogramme analysés dans notre étude figurent dans le tableau I.

L'analyse des valeurs de la leucospermie montre que la pyospermie (G1) était présente dans 5,88\% des spermes (49/833). G2 était composé d'environ la moitié des spermes (414/833). La répartition des spermes selon la 
leucospermie est donnée dans la figure 1.

La comparaison des valeurs moyennes des paramètres du sperme entre les deux groupes ne montre pas de différences significatives (Tableau II).

Cependant, l'oligospermie était plus fréquente dans G1 (Tableau IV). A l'inverse, la tératospermie (formes anormales $>70 \%$ ) était plus fréquente dans G2. L'asthénospermie et la nécrospermie étaient très fréquentes dans les deux groupes (Tableau IV).

Il n'existait pas de corrélations significatives entre les valeurs de la leucospermie et les paramètres du spermogramme (Tableau III).

Les distributions des paramètres du sperme en fonction de la leucospermie sont données dans les figures $2,3,4,5$ et 6 .

L'étude rétrospective des dossiers des patients pyospermiques a révélé l'existence dans $55 \%$ des cas (22/40), d'au moins un signe évocateur de l'infection génitale (aspect trouble du sperme, sérologie anti-chlamydienne positive, spermoculture positive et anomalies du plasma séminal ( $\mathrm{pH}$ élevé et temps de liquéfaction supérieur à 2 heures).

\section{DISCUSSION}

La présence des leucocytes dans le sperme témoigne habituellement d'un état inflammatoire chronique non spécifique des voies génitales. Un nombre élevé de leucocytes ou pyospermie peut être associé à l'infertilité masculine et peut traduire l'existence d'une infection génitale [7].

La prévalence de la pyospermie est estimée comprise entre 10 et $20 \%$ chez les hommes consultant pour infertilité du couple [18, 24]. Cependant, dans notre étude la pyospermie était plus rare $(5,88 \%)$. Kung [22] et Sigman [30] ont rapporté des prévalences plus faibles, $2 \%$ et $3,5 \%$ respectivement.

Il est admis que les leucocytes proviennent des voies excrétrices séminales et surtout des glandes annexes, vésicules séminales et prostate [23, 30]. Cependant, récemment, Simbini et coll ont démontré que les leucocytes étaient plus prévalents dans la première et la deuxiè- me fraction du sperme que dans la troisième fraction et ont conclu que le testicule, l'épididyme et la prostate sont les sources majeures des leucocytes séminaux et que la contribution des vésicules séminales est minime [31].

Les conséquences habituellement admises d'une importante leucospermie sont une baisse de la numération, une mobilité progressive et hyperactivée diminuées et une fonction de fusion du spermatozoïde perturbée. Cependant, des controverses existent sur les conséquences de la leucospermie sur la qualité du sperme et sur les capacités fécondantes des spermatozoïdes. Malgré ces controverses il a été bien démontré in vitro que les leucocytes exercent un effet délétère sur les capacités fonctionnelles des spermatozoïdes par l'intermédiaire des dérivés actifs de l'oxygène (DAO) $[1,17]$. Ces DAO s'attaquent aux acides gras polyinsaturés des phospholipides membranaires entraînant des réactions de peroxydation lipidique qui se propagent de proche en proche et provoquent la désorganisation de l'architecture moléculaire et une baisse de la fluidité membranaire [5].

Dans notre étude, nous n'avons pas trouvé de différences significatives entre les valeurs moyennes des principaux paramètres du spermogramme en présence ou en absence de pyospermie. Gonzales [16] n'a pas non plus trouvé dans son étude portant sur 280 hommes infertiles de différence concernant la numération, la mobilité ou la morphologie des spermatozoïdes en fonction de la leucospermie.

Dans l'étude de Simbini et coll [31], Il existait une relation inverse entre le nombre de leucocytes et la numération des spermatozoïdes. Le pourcentage des spermatozoïdes de forme anormale était plus élevé et la mobilité plus faible en cas de leucospermie. Des résultats similaires ont été rapportés par Yanushpolsky [34] et Wolff [35]. Par ailleurs, il a été rapporté que la leucospermie affectait la vitalité des spermatozoïdes et la composition du plasma séminal en protéines (augmentation de l'albumine et diminution des marqueurs prostatiques) $[11,13]$.

Bien que dans notre étude il n'existait pas de corrélation significative entre les valeurs de la 
Tableau I : Valeurs moyennes \pm écart-types des principaux paramètres dans les 833 spermes.

\begin{tabular}{|c|c|c|}
\hline PARAMETRES & $\begin{array}{l}\text { MOYENNE } \pm \\
\text { ECART TYPE }\end{array}$ & NORMES \\
\hline Volume (ml) & $3,4 \pm 2,4$ & $2-6$ \\
\hline Mobilité (\%) & $40 \pm 14,3$ & $\geq 50$ \\
\hline $\begin{array}{l}\text { Numération } \\
(106 / \mathrm{ml})\end{array}$ & $79,2 \pm 141,4$ & $\geq 20$ \\
\hline Vitalité $(\%)$ & $59,5 \pm 18,2$ & $\geq 75$ \\
\hline $\begin{array}{l}\text { Morphologie } \\
\text { (\% formes normales) }\end{array}$ & $33,6 \pm 15,4$ & $\geq 30$ \\
\hline
\end{tabular}

Tableau II : Valeurs moyennes et écart types des principaux paramètres du sperme dans les deux groupes de patients avec (G1) et sans pyospermie (G2).

\begin{tabular}{|c|c|c|c|c|}
\hline PARAMETRES & $\begin{array}{c}G 1 \\
(n=49) \\
\end{array}$ & $\begin{array}{c}\text { G2 } \\
(n=414) \\
\end{array}$ & $\begin{array}{c}\text { NORMES } \\
\text { (OMS) }\end{array}$ & $\begin{array}{c}\text { TEST } \\
\text { STATISTIQUE }\end{array}$ \\
\hline Volume (ml) & $3,3 \pm 1,5$ & $3,6 \pm 3,0$ & $2-6$ & NS \\
\hline Mobilité (\%) & $39,6 \pm 16,7$ & $39,9 \pm 13,5$ & $\geq 50$ & NS \\
\hline Numération $(106 / \mathrm{ml})$ & $69,4 \pm 86,2$ & $68,3 \pm 77,8$ & $20-200$ & NS \\
\hline Leucocytes $(106 / \mathrm{ml})$ & $2,3 \pm 1,5$ & $0,2 \pm 0,1$ & $<1$ & \\
\hline Vitalité (\%) & $57,7 \pm 20,0$ & $59,6 \pm 17,8$ & $\geq 75$ & NS \\
\hline Morphologie (\%) & $35,8 \pm 13,0$ & $33,2 \pm 15,6$ & $\geq 30$ & NS \\
\hline
\end{tabular}

Tableau III : Coefficients de corrélation ( $r$ ) entre la leucospermie et les différents paramètres du sperme chez l'ensemble des patients.

\begin{tabular}{lccccc}
\hline $\begin{array}{l}\text { PARAMETRES } \\
\text { DU SPERME }\end{array}$ & VOLUME & NUMERATION & MOBILITE & MORPHOLOGIE & VITALITE \\
\hline $\begin{array}{l}\text { Coefficient de } \\
\text { corrélation avec la } \\
\text { leucospermie }\end{array}$ & $-0,007$ & 0,02 & $-0,01$ & 0,06 & $-0,02$ \\
\hline
\end{tabular}

Tableau IV : Fréquences des différentes anomalies du sperme dans les groupes G1 et G2.

\begin{tabular}{lccc}
\hline ANOMALIE & G1 & G2 & TEST STATISTIQUE \\
\hline Azoospermie & $\%$ & $\%$ & NS \\
Oligospermie & 8,9 & 6,1 & $\mathrm{~S}(\mathrm{p}<0,01)$ \\
Asthénospermie & 20,3 & 40,8 & $\mathrm{NS}$ \\
Tératospermie & 79,2 & 71,5 & $\mathrm{~S}(\mathrm{p}<0,05)$ \\
Nécrospermie & 47,2 & 34,1 & $\mathrm{NS}$ \\
\hline
\end{tabular}




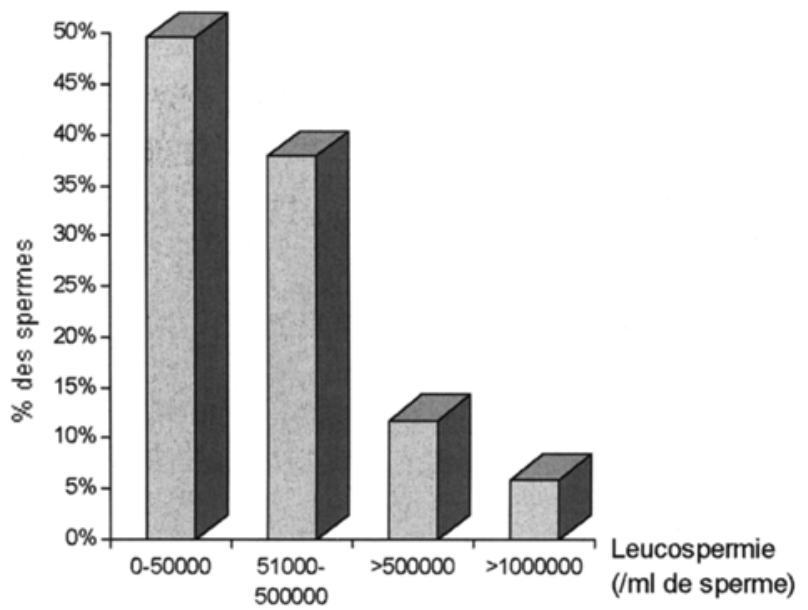

Figure 1 : Répartition des spermes selon la leucospermie chez tous les patients infertiles.

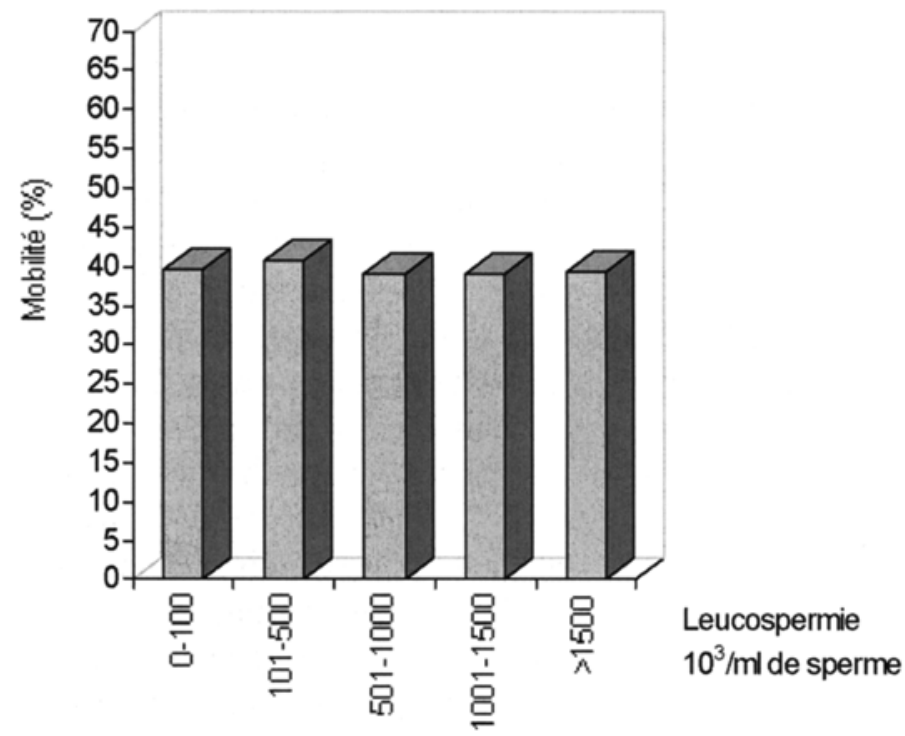

Figure 3 : Répartition détaillée de la mobilité en fonction de la leucospermie dans tous les spermes.

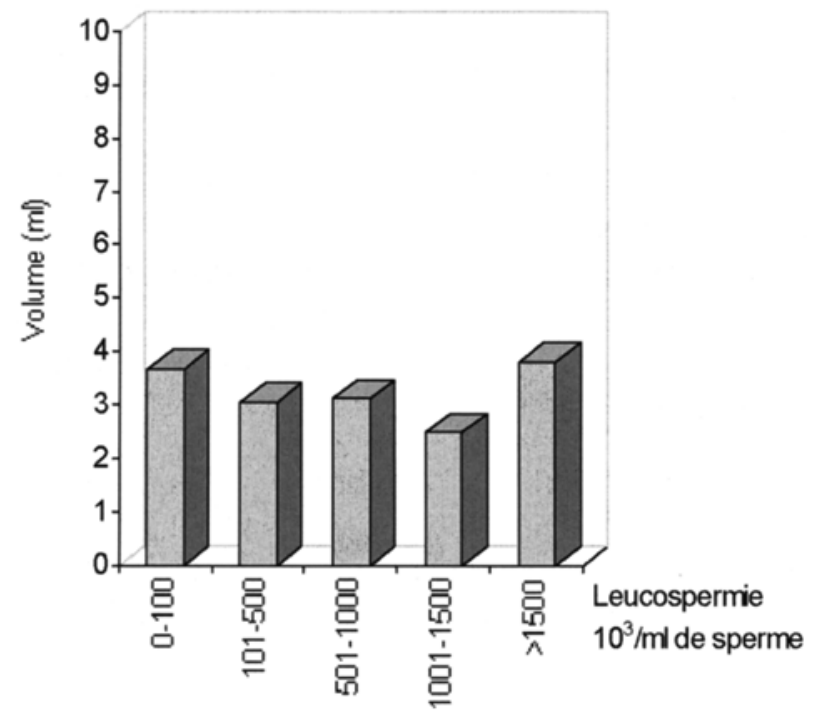

Figure 5 : Répartition du volume du sperme en fonction de la leucospermie chez tous les patients.

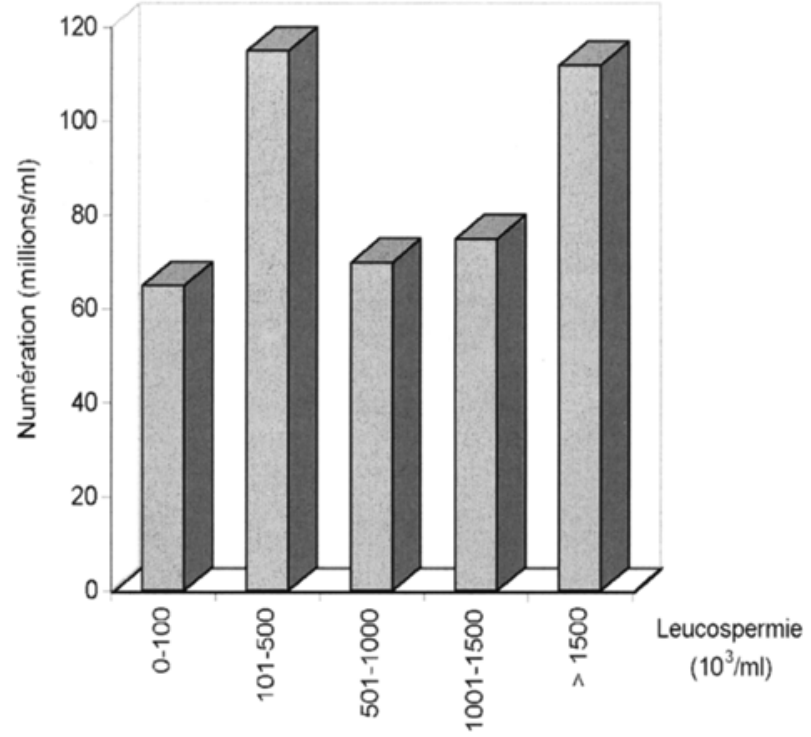

Figure 2 : Répartition des valeurs moyennes de la numération des spermatozoïdes en fonction de la leucospermie chez l'ensemble des patients.

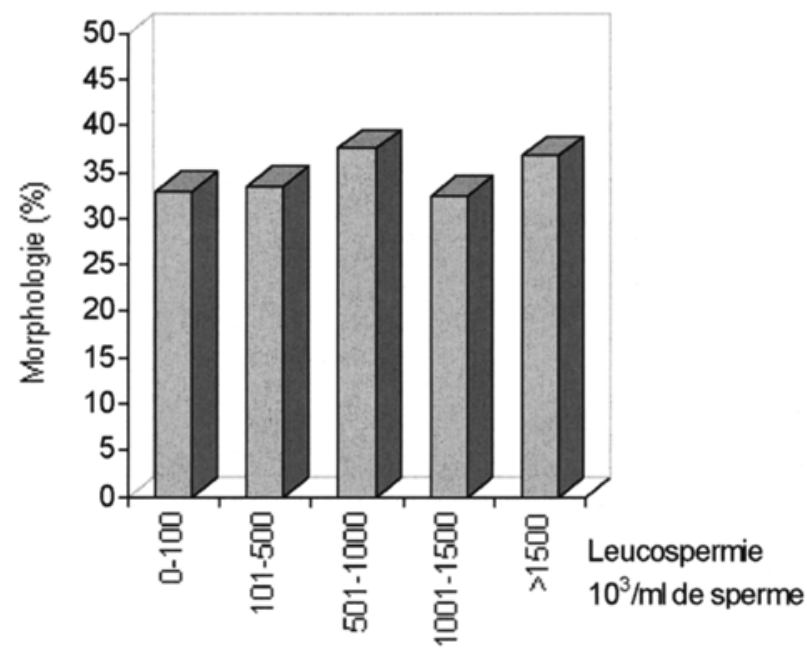

Figure 4 : Répartition de la morphologie en fonction de la leucospermie chez l'ensemble des patients.

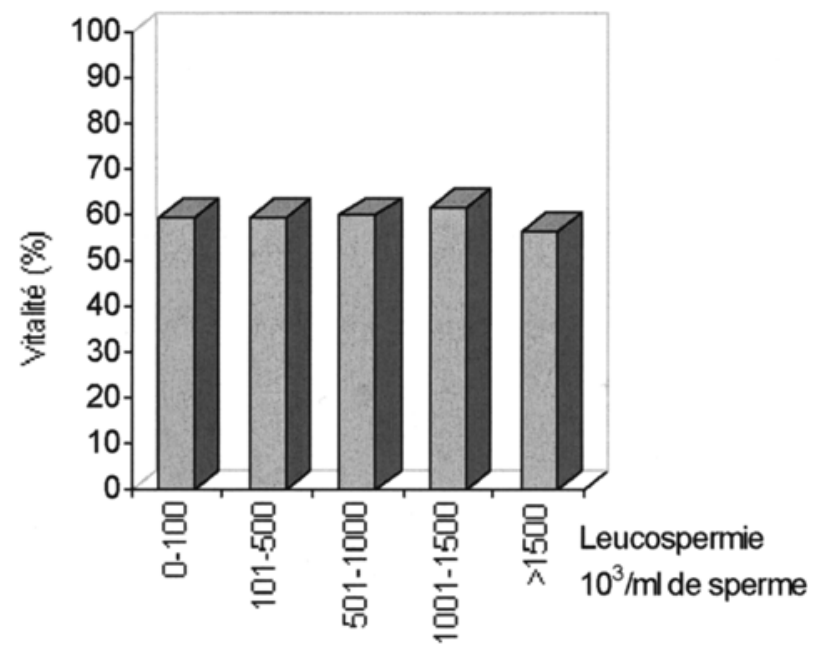

Figure 6 : Répartition de la vitalité en fonction de la leucospermie pour les 833 spermes. 
leucospermie et la morphologie, la fréquence de la tératospermie (taux anormalement élevé de spermatozoïdes de forme anormale) était significativement plus faible chez les patients pyospermiques, ce qui pourrait traduire un rôle des leucocytes dans l'élimination par phagocytose des spermatozoïdes anormaux. Cette hypothèse a été vérifiée dans une étude ancienne par Berger et coll [9]. Kiessling et coll [20] ont observé un plus grand nombre de spermatozoïdes de morphologie normale dans les spermes contenant plus de deux millions de cellules rondes par millilitre avec prédominance de leucocytes. Tomlinson et coll [32] ont trouvé une corrélation positive entre la concentration des leucocytes et le taux de spermatozoïdes à forme normale. Ils ont aussi conclu que les leucocytes interviennent probablement pour éliminer les spermatozoïdes anormaux.

Menkveld et coll [26] ont montré que la morphologie des spermatozoïdes était modifiée lorsque le sperme présentait une augmentation des polynucléaires, en particulier des allongements des têtes. Kung [22] a trouvé une corrélation inverse entre le taux de spermatozoides de forme normale et le nombre de lymphocytes $\mathrm{T}$ helper détectés par des anticorps monoclonaux. Des résultats contradictoires ont été rapportés dans d'autres études [32, 31, 16].

L'autre anomalie constatée dans notre étude est l'oligospermie qui était plus fréquente dans le groupe de patients avec pyospermie. Cette constatation appuie l'hypothèse selon laquelle l'inflammation du tractus génital, associée ou non à l'infection entraîne des remaniements histologiques aboutissant à une réduction de la perméabilité des voies génitales [27], ou encore à une perturbation de la fonction gamètogène. Nous avons nous même démontré dans une étude précédente [2] que l'infection génitale à Chlamydia trachomatis était associée à une fréquence élevée de l'oligospermie et nous avons suggéré que ce résultat était en faveur de l'atteinte testiculaire et/ou des voies spermatiques qui seraient partiellement obstruées par des remaniements tissulaires secondaires à la réaction inflammatoire engendrée par Chlamydia trachomatis.

Kortebani et coll [21] ont trouvé que l'asthénospermie était l'anomalie du sperme la plus fré- quente chez les patients infertiles avec pyospermie. Dans notre étude, bien que l'asthénospermie était très fréquente, il n'existait pas de différence significative entre les deux groupes de patients avec et sans pyospermie.

Dans notre étude, nous n'avions pas suffisamment d'arguments cliniques et biologiques pour établir avec précision un éventuel lien entre la pyospermie et l'infection génitale. Cependant, nous avons démontré précédemment [2] que la leucospermie n'était pas différente entre les patients positifs à un ou plusieurs marqueurs (PCR, Immunofluorescence directe, Elisa, culture cellulaire...) de l'infection à Chlamydia trachomatis et les patients sans infection chlamydienne. Ceci pourrait suggérer que le germe en cause déclenche plutôt une réaction immunitaire qui mobilise des lymphocytes $\mathrm{T}$ et/ou $\mathrm{B}$ et n'entraîne pas un afflux de granulocytes comme dans les infections à germes banals. Dans d'autres études, il a été montré que le nombre de leucocytes (peroxydase positive) était bien corrélé avec la bactériospermie [19] et que lors des prostatites chroniques et les urétrites, le nombre des granulocytes ainsi que l'élastase (enzyme spécifique des polynucléaires) étaient significativement augmentés [25, 8]. De même, chez les patients infertiles avec leucospermie, les signes cliniques d'infection urinaire (dysurie, hémospermie....) étaient significativement plus fréquents qu'en l'absence de leucospermie [11].

Bien que la leucospermie soit le reflet d'une inflammation et/ou d'une infection du tractus génital, il ne semble pas que la présence des leucocytes affecte les fonctions des glandes annexes. En effet, Saad et coll [29] ainsi que Simbini et coll [31], n'ont pas constaté de diminution des marqueurs biochimiques épididymaires ( $\alpha$-glucosidase), prostatiques (acide citrique) et vésiculaires (fructose) en rapport avec la leucospermie.

Il ne semble pas, par ailleurs, que la leucospermie soit associée à une réaction immunitaire locale, puisque Habermann [18] et coll n'ont pas trouvé de différence de nombre de leucocytes entre les patients avec ou sans anticorps anti-Chlamydia dans le sperme. Cependant, dans l'étude de Paschke [28] il y avait une incidence élevée des leucocytes $(\geq 1$ 
million/ml de sperme) chez les patients avec Mar-test positif (IgG et IgA). Selon EggerKruse [13], les leucocytes pourraient être attirés vers le tractus génital en réponse à la présence de débris cellulaires.

Concernant le retentissement de la pyospermie sur les capacités fécondantes des spermatozoïdes, Tomlinson et coll [32] ont montré contrairement à d'autres auteurs [15, 33, 9] que la présence de leucocytes dans l'éjaculat n'affectait pas les résultats de la FIV. Ils ont montré aussi une corrélation positive entre la concentration des leucocytes et le taux de conception.

De point de vue thérapeutique, la prescription d'antibiotiques chez les hommes présentant une pyospermie avec ou sans preuve d'infection, semble améliorer de manière significative la qualité du sperme et ses capacités fécondantes. En effet, dans une étude portant sur des couples avec infertilité inexpliquée, Branigan et coll [10] ont rapporté que 53\% des épouses des hommes pyospermiques devenaient enceintes dans les 6 mois après une antibiothérapie, contre seulement $6 \%$ chez les femmes des hommes non traités. Par ailleurs, le traîtement in vitro des spermes par des antioxydants réduit les effets délétères des polynucléaires activés sur la mobilité [6].

En conclusion, nous avons montré dans notre étude que la prévalence de la pyospermie était faible chez les hommes infertiles et que cette anomalie du sperme ne semble pas altérer les paramètres morphologiques et fonctionnels du spermogramme. Cependant, la présence de nombreux leucocytes, reflet d'un état inflammatoire du tractus génital, était associée à une fréquence élevée de l'oligospermie ; celle-ci pourrait être la conséquence d'une diminution de la perméabilité des voies spermatiques ou de l'atteinte histologique des testicules. Paradoxalement, la pyospermie était associée à une réduction de la tératospermie traduisant probablement un rôle favorable des leucocytes sur l'élimination de cellules germinales anormales. Aucune relation n'a pu être établie dans cette étude rétrospective entre la leucospermie et l'infection génitale, du fait de l'insuffisance d'arguments cliniques et biologiques. Il serait donc utile de la compléter par une étude pros- pective comportant une exploration exhaustive avec recherche systématique de germes et des anticorps anti-spermatozoïdes ainsi que le dosage des marqueurs biochimiques du sperme. L'objectif d'une telle étude sera de déterminer avec précision le lien existant entre la leucospermie et l'infection et le retentissement de la pyospermie sur l'activité des différents compartiments glandulaires du tractus génital masculin et enfin de chercher une éventuelle relation avec une réaction immunitaire locale.

\section{REFERENCES}

1. AITKEN R.J., WEST K., BUCKINGHAM D. : Leukocytic infiltration into the human ejaculate and its association with semen quality, oxidative stress, and sperm function. J. Androl., 1994, 15 : 434-452.

2. AMMAR-KESKES L., GDOURA R., BOUZID F. et al : Retentissement de l'infection génitale à Chlamydia trachomatis sur le sperme chez les hommes consultant pour infertilité du couple. Andrologie, 1998, 8 , 25-35.

3. AMMAR-KESKES L., KALLEL N., BOUZID F,, REKIK S., REBAÏ T. : Caractéristiques cyto-morphologiques du sperme chez les hommes consultant pour infertilité du couple dans la région de Sfax. Andrologie, 1998, 8, 287-301.

4. AUGER J., JOUANNET P. : Valeurs de référence des caractéristiques du sperme. In : AugerJ., Jouannet P., Ed INSERM. Manuel de laboratoire de l'OMS. Analyse du sperme humain et de l'interaction des spermatozoïdes avec le mucus cervical, Paris, 1993 :45-46.

5. AUGER J. : Dérivés actifs de l'oxygène et dysfonctions spermatiques : rôle de l'infection du tractus génital de l'homme?. Andrologie, 1998, 8, nº3 : 234-244.

6. BAKER HW., BRINDLE J., IRVINE DS., AITKEN RJ. : Protective effect of antioxydants on the impairement of sperm motility by activated polymorphonuclear leukocytes. Fertil. Steril., 1996, 65, 2: 411-9.

7. BAR-CHAMA N., FISCH H. : Infection and pyospermia in male infertility. World J. Urol., 1993, 11(2) :7681.

8. BARRAT C., HARRISON P., ROBINSON A., KESSOPOULOU E.: Seminal white cells in men with urethral tract infection. A monoclonal antibody study. British J. Urol., 1991, 68 : 531-536.

9. BERGER RE., KARP LE., WILLIAMSON RA., KOEHLER J., MOORE DE., HOLMES KK. : The relationship of pyospermia and seminal fluid bacteriology to sperm function as reflected in the sperm penetration assay. Fertil. Steril., 1982, $37: 557-564$.

10. BRANIGAN EF., SPADONI LR., MULLER CH. : 
Identification and treatement of leukocytospermia in couples with unexplained infertility. J Reprod. Med., 1995, 40, 9: 625-9.

11. COLPI GM., ROVEDA ML., TOGNETTI A., BALERNA M. : Seminal tract inflammation and male infertility. Correlations between leukospermia and clinical history, prostatic cytology, conventional semen parameters, sperm viability and seminal plasma protein composition. Acta. Eur. Fertil., 1988, 19, 2: 69-77.

12. EGGERT-KRUSE W., BELLMAN A., ROHR G., TILGEN W., RUNNEBAUM B. : Differentiation of round cells in semen by means of monoclonal antibodies and relationship with male fertility. Fertil. Steril., 1992, 58, 5: 1046-55.

13. EGGERT-KRUSE W., PROBST S., ROHR G., AUFENANGER J., RUNNEBAUM B. : Screening for subclinical inflammation in ejaculates. Fertil. Steril., 1995, 64 , 5: 1012-1022.

14. FEDDER J., ASKJAER SA., HJORT T. : Nonspermatozoal cells in semen : relationship to other semen parameters and fertility status of the couple. Arch. Androl., 1993, 31, 2: 95-103.

15. FEDDER J. : Nonsperm cells in human semen : with special référence to seminal leukocytes and their possible influence on fertility. Arch. Androl., 1996, 36, 1: 41-65.

16. GONZALES GF., KORTEBANI G., MAZZOLI AB. : Leucocytospermia and function of the seminal vesicules on seminal quality. Fertil. Steril, 1992, 57, 5: 1058-1065.

17. GRIVEAU J.F., LE LANNOU D. : Radicaux libres et spermatozoïdes humains : physiologie et physiopathologie. Andrologie, 1995, 5 : 369-381.

18. HABERMANN B., KRAUSE W. : Altered sperm function or sperm antibodies are not associated with chlamydial antibodies in infertile men with leucocytospermia. J. Eur. Acad. Dermatol. Venerol., 1999, 12, 1: 259.

19. JEDRZEJCZAK P., SZUMALA-KAKOL A, DYDOWICZ P., SZYMANOWSKI K., PISARSKI T.: Usefulness of couting leukocytes and round cells in determination of bacterial infection of semen in infertile men. Ginekol. Pol. 1996, 67, 11: 569-73.

20. KIESSLING AA., LAMPARELLI N., YIN HZ., SEIBEL MM., EYRE RC. : Semen leukocytes : friends or foes ? Fertil. Steril., 1995, 64 : 196-198.

21. KORTEBANI G., GONZALES GF., BARRERA C., MAZZOLLI AB. : Leucocyte populations in semen and male accessory gland function : relationship with antisperm antibodies and seminal quality. Andrologia, 1992, 24, 4: 197-204.

22. KUNG AW., HO PC., WANG C. : Seminal leucocyte subpopulations and sperm function in fertile and infertile Chinese men. Int. J. Androl. 1993, 16, 3 : 18994.

23. LANGE A., PONGILUPPI F., GIUDICI M., BORELLA T. : Cytologic diagnosis of azoospermia. Minerva Med, 1983, 74 : 1423-1426.

24. LUCAS H. : Inflammation de l'appareil génital masculin et reproduction : traitement in vitro. Andrologie, $1998,8, n^{\circ} 3: 269-279$.

25. LUDWIG G., KUMMEL C., SCHROEDERPRINTZIN I., RINGERT RH., WEIDNER W. : Evaluation of seminal parameters in patients with chronic prostatis or leucospermia. Andrologia, 1998, $30: 41-47$.

26. MENKVELD R., KRUGER TF. : Sperm morphology and male urogenital infections. Andrologia, 1998, 30 : 49-53.

27. MIEUSSET R. : Inflammation de l'appareil génital masculin et reproduction : traitement in vivo. Andrologie, 1998, $8: 259-268$.

28. PASCHKE R., SCHULZE BERTELSBECK D., BAHRS S., HEINECKE A., BEHRE HM. : Seminal sperm antibodies exhibit an unstable spontaneous course and an increased incidence of leucocytospermia. Int. J Androl., 1994, 17, 3: 135-9.

29. SAAD A., BEN ALI H., RHIMI Z., ZEMNI S., KHAIRI H., BOUZAKOURA C. : La leucospermie a-t-elle une influence sur l'état épididymaire apprécié par le dosage de l'alpha 1-4 glucosidase ?. Contracep.Fertil. Sex., 1990, 18, 2 :1107-1110.

30. SIGMAN M., LOPES L. : The correlation between round cells and white blood cells in the semen. J. Urol., 1993, 149 : 1338-40.

31. SIMBINI T., UMAPATHY E., JACOBUS E., TENDAUPENYU G., MBIZVO MT. : Study on the origin of seminal leucocytes using split ejaculate technique and the effect of leucocytospermia on sperm characteristics. Urol. Int., 1998, 61, 2 : 95-100.

32. TOMLINSON MJ., BARRATT CL., COOKE ID. : Prospective study of leukocytes and leukocyte subpopulations in semen suggests they are not a cause of male infertility. Fertil. Steril., 1993, 60, 6: 1069-75.

33. VAN DER VEN HH., JEYENDRAN RS., PEREZPELAEZ M., AL-HASANI. S., DIEDRICH K., KREBS D. : Leucospermia and the fertilizing capacity of spermatozoa. Eur J Obstet Reprod Biol 1987, 24(1) : 49-52.

34. YANUSHPOLSKY EH., POLITCH JA., HILL JA., ANDERSON DJ. : Is leukocytospermia clinically relevant ? Fertil. Steril., 1996, 66, 5 : 822-5.

35. WOLFF H., POLITCH JA., MARTINEZ A., HAIMOVICI F., HILL JA., ANDERSON DJ. : Leukocytospermia is associated with poor semen qua- 
lity. Fertil. Steril., 1990, 53, $3:$ 528-36.

\author{
ABSTRACT \\ Prevalence of pyospermia and impact on \\ semen parameters in infertile men \\ L. AMMAR-KESKES, N. CHAKROUN-FKI, \\ F. BOUZID, A. BAHLOUL, S. MASMOUDI, \\ M. TRIFA, T. REBAII, S. REKIK, \\ N. MHIRI.
}

There are numerous controverses concerning the relationship between the presence of leukocytes in semen and male infertility. In the aim to determine the impact of pyospermia on sperm quality, we have realised a retrospective study in which we analysed and compared semen parameters and abnormalities frequencies between pyospermic and non pyospermic infertile patients.

833 spermiograms were included in this study. They were done in accord with WHO method. Leucocytes identification was performed by cyto-enzymologic method that reveals myelo-peroxydase in polymorphonuclear granulations. Pyospermia was considered when number of leucocytes was more than one million per millilitre of sperm. The non pyospermic group was composed by sperm with leucocytospermia less than $\mathbf{5 0 . 0 0 0}$ per millilitre.

The prevalence of pyospermia was $5.88 \%$. There was not significative difference of semen parameters (volume, motility, morphology, number of spermatozoa and viability) between pyospermic and non pyospermic groups. In the other hand, no correlation was found between leucospermia and semen parameters. However, oligospermia was significantly more frequent in pyospermic group $(40.8 \%)$ than in non pyospermic group $(20,3 \%)$. Inversly, the frequence of teratospermia was significantly higher in non pyospermic group $(47.2 \%$ vs $34.1 \%, p<0,05)$. These results suggest that inflammation and/or infection associated with pyospermia is complicated by reduction of spermatic ducts permeability. Although, the leucocytes would act in removing amorphous gametes by phagocytosis. No relationship was established between pyospermia and infection. In future, a prospective study would be done with exhaustive exploration of pyospermia etiology, with hopes to clarify the true link with infection and autoimmune reaction and to determine the effect of pyospermia on glandular activities in male genital tract and on functionnal properties of spermatozoa.

Key Words : Pyospermia, leukospermia, spermiogram, spermatozoa, male infertility, inflammation. 